\title{
An iterative Approach to Deriving Drug Infusions
}

\author{
David P. Crankshaw \\ Department of Pharmacology, The University of Melbourne, Melbourne, Victoria, Australia \\ Email: dpcran@unimelb.edu.au \\ Received 2012
}

\begin{abstract}
A simple iterative process can be used to generate intravenous drug infusion profiles. It overcomes limitations in deriving compartmental pharmacokinetic models and has application to evaluation of new drugs and to clinical practice.
\end{abstract}

Keywords: Pharmacokinetics; Drug Delivery; Intravenous Infusion

\section{Introduction}

Compartmental pharmacokinetic models (CPM) commonly of three compartments together with inter-compartmental rate constants are usually derived from data obtained during the elimination phase following administration of a single dose of a drug. The CPM is widely used to predict the intravenous infusion rate for delivery of hypnotics and opioids during anaesthesia and in intensive therapy units. Assumptions are made that the CPM accurately describes the distribution and elimination of the drug during infusion as predicted from a single dose. This approach has proved effective in clinical situations where the operator is free to adjust the target concentration according to clinical impressions of dosing requirements [1]. However, it has been observed that drug levels based on predictions from a CPM commonly result in higher blood levels. This appears to be due to an over estimation of the central compartment $\left(\mathrm{V}_{1}\right)$ together with an over estimation of the rate of elimination [2]. In a widely used commercial application of the CPM, an arbitrary reduction in the size of $\mathrm{V}_{1}$ was incorporated to overcome this problem [3]. Such an approach to programmed delivery, while expedient and clinically effective for drugs with a large therapeutic index, has considerable limitations if used in a rigorous way for experimental or regulatory purposes.

Further points are that therapeutic agents may have considerable effect on the cardiovascular system resulting in concentration dependent changes in distribution and elimination. In particular, the cardiac output may differ at different concentrations and directly or indirectly influence the flow of drug to various organs of the body particularly the liver and kidneys. Further, the metabolism of drugs may be concentration dependent. If a CPM is derived during the elimination phase following a single bolus dose, cardiac output may be considerably higher than at therapeutic levels during an infusion. Similarly, if drug metabolism is concentration dependent, a higher rate of metabolism at lower concentrations may lead to an over estimation of infusion requirements when concentrations are higher.

Population pharmacokinetic modeling such as STANPUMP [4] using NONMEM software [5] can be used to create models that are concentration dependent. But implementation requires specialized knowledge, is computationally intense, time con- suming and complex.

We have described a method which requires no more than simple arithmetic to create a table of values infusion rates against time suitable for manual implementation with a standard syringe pump [6]. We have termed this iterative method for developing infusion profiles at specific predetermined concentrations the Plasma Drug Efflux (PDE) method. The method has been described by us for a range of drugs infused into human patients during the conduct of clinical anaesthesia [6-8].

\section{Methods}

Following published methodology [6] an arbitrary fixed rate infusion of the drug under investigation is administered to the first individual as a continuous fixed rate infusion with dosage based on total body mass (TBM) or estimated lean body mass (LBM). Arterial blood is sampled at frequent intervals from the commencement until the end of the infusion period and assayed for total blood concentration $\left(\mathrm{C}_{\mathrm{m}}\right)$.

The intravenous infusion site is considered the reference point so each measured arterial blood concentration is corrected by an arbitrary value of minus one minute to allow for vein to artery transit time. The delivery rate (mg. $\mathrm{min}^{-1} \cdot \mathrm{kg}_{\mathrm{LBM}}{ }^{-1}$ ) is then divided by the time corrected arterial plasma concentration (mg.L $\mathrm{L}^{-1}$ ). This calculation is successively applied to all concentration measurements during the study period in order to determine the Plasma Drug Efflux values (Ep) (L.min ${ }^{-1} \cdot \mathrm{kg}_{\mathrm{LBM}}{ }^{-1}$ ). The values for the first subject are plotted as a function of time and a set of values of Ep at one minute intervals is generated by linear interpolation. These values were transferred to a programmable infusion device [8]. An infusion is then administered to a second group of around two subjects with a nominated target concentration $\left(\mathrm{C}_{\mathrm{TGT}}\right)$. The delivery rate (mg.min ${ }^{-1}$ ) for each subject is produced by continuous multiplication of successive values of Ep (read from the memory each minute) by $\mathrm{C}_{\mathrm{TGT}}\left(\mathrm{mg} . \mathrm{L}^{-1}\right)$ and by the TBM or LBM (kilogram) of the particular subject. A table may be produced for a range of subject weights and target concentrations and the infusion implemented by manual setting of a standard infusion pump at times obtained from the table [9]. 
Table I. Bolus doses (ml) and infusion rate, in seven steps from zero to $150 \mathrm{~min}$, derived from published data of thiopental [7], methohexital [7], alfentanil [8] and propofol [6] in order to achieve a clinically appropriate target concentration in an anaesthetized subject of 50kg LBM.

\begin{tabular}{|c|c|c|c|c|c|c|c|c|c|c|}
\hline & & & \multicolumn{8}{|c|}{ Infusion period (min) } \\
\hline & & & $0-5$ & $5-10$ & $10-20$ & $20-30$ & $30-60$ & $60-90$ & $90-120$ & $120-150$ \\
\hline Drug & Target conc. (mg. $\mathrm{L}^{-1}$ ) & Bolus (ml) & & & & Infusion & rate $(\mathrm{ml}$ & & & \\
\hline $\begin{array}{l}\text { Thiopental } \\
\left(25 \mathrm{mg} \mathrm{ml}^{-1}\right)\end{array}$ & 10 & 4.0 & 38 & 26 & 19 & 16 & 14 & 13 & 12 & 11 \\
\hline $\begin{array}{l}\text { Methohexital } \\
\left(10 \mathrm{mg} \mathrm{ml}^{-1}\right)\end{array}$ & 5 & 5.0 & 57 & 46 & 38 & 32 & 28 & 26 & 25 & 24 \\
\hline $\begin{array}{l}\text { Alfentanil } \\
\left(0.5 \mathrm{mg} \cdot \mathrm{ml}^{-1}\right)\end{array}$ & 0.1 & 0.87 & 15 & 10 & 6 & 4 & 3 & - & - & - \\
\hline $\begin{array}{l}\text { Propofol } \\
\left(10 \mathrm{mg} \cdot \mathrm{ml}^{-1}\right)\end{array}$ & 3 & 4.3 & 66 & 57 & 47 & 42 & 42 & 42 & 42 & 42 \\
\hline
\end{tabular}

Successive groups of subjects are then infused using same process with the infusion rate-time profile calculated from the immediately previous group of subjects. The size of each group is chosen to increase progressively during the iterative process. The decision to accept an infusion profile as optimal and stop the iterative process can be made by comparing values of $C_{m}$ at each sampling point using Wilcoxon's matched pairs test (CSS-Statsoft) to identify bias from $\mathrm{C}_{\mathrm{TGT}}$. A non-significant result $(\mathrm{P}>0.05)$ is used to terminate the iterative process.

When determining the Ep profile for propofol, groups of 1, 2, 5 and 11 were used to obtain a suitable end point [6].

\section{Results}

Previously published data for thiopental [7], methohexital [7], alfentanil [8] and propofol [6] has been used to develop bolus and infusion rates for each drug. Table I shows an application of such data in a form suitable for clinical implementation. An initial bolus dose and subsequent infusion rates, as a series of steps suitable for implementation with a simple syringe pump, are shown for a patient of $50 \mathrm{~kg}$ lean body mass. For further flexibility, a simple spread sheet could be based on these data to develop data for a range of patient weights and a range of target concentrations.

\section{Discussion}

This iterative process has been used to determine infusion requirements for a number of drugs commonly used in anaesthetic practice. The method is highly efficient in proceeding towards a defined endpoint. The values of Plasma Drug Efflux (Ep) represent, at a constant plasma concentration, the expected time varying, rate of loss of drug from the site of intravenous injection in units of drug clearance. The values represent "clearance" from the intravenous site of injection whether the loss is by redistribution, binding to tissues, metabolism, or elimination from any organ.

Data, obtained with this time and resource efficient method, can be processed with NONMEM to yield standard CPM parameters (see [6]) and are of immediate practical use in a number of ways.

A pharmacokinetic model generated in this way is free of the major deficiencies of standard methods which fail to define the concentration at which the model is determined. Models derived over a range of concentrations, for drugs with significant cardiovascular effects, are in fact not accurate at any concentration.

If the PDE method is used in a series of studies over a range of concentrations, effects of the drug under well controlled conditions can be observed. Data from this approach can then be used in the design of more accurate preprogrammed infusion devices.

The method can also be used to determine optimal drug infusion profiles for a wide range of drugs in both animal and human subjects. This has application in drug development.

Constant drug levels can be used to investigate the actions of drugs on a wide range of organ systems.

Finally, the efficiency of the method allows researchers to investigate variability due to species, age, gender and race as well as drug interactions.

\section{REFERENCES}

[1] P. S. A. Glass, S. L. Shafer and J. G. Reeves, "Intravenous drug delivery systems,” In Miller’s Anesthesia, vol. 1, R. G. Miller Ed. Churchill Livingstone Elsevier, 2010, pp. 826-830.

[2] J. A. H. Davidson, A. D. Macleod, J. C. Howie, M. White, G. N. C. Kenny, "Effective concentration 50 for propofol with and without 67\% nitrous oxide,” Acta. Anaesthesiol. Scand. Vol. 37, pp. 458-464, 1993.

[3] B. Marsh, M. White, N. Morton and G. N. C. Kenny, "Pharmacokinetic model driven infusion of propofol in children," Anesth. Analg. Br. J. Anaesth. vol. 67, pp. 41-48, 1991.

[4] Stanpump ${ }^{\circledR}$ (Steven L. Shafer: Stanford University Anesthesiology Service, 112A PAVAMC, 3801 Miranda Ave, Palo Alto, CA 94304, USA).

[5] S. L. Beal, L. B. Scheiner, “NONMEM User's Guide,” San Francisco, University of California San Francisco, 1979.

[6] D. P. Crankshaw, M. J. Brown and A. R. Bjorksten, “A comparison of parametric and non-parametric approaches to target-controlled infusion of propofol,” Anaesth. Intensive Care vol. 38, pp. 437-444, May 2010.

[7] D. P. Crankshaw, M. D. Boyd, A. R. Bjorksten, "Plasma Drug Efflux - A new approach to optimization of drug infusion for constant blood concentration of thiopental and methohexital," 
Anesthesiology, vol. 67 pp. 32-41, 1987.

[8] D. P. Crankshaw, D. J. Morgan, G. H. Beemer and F. Karasawa, "Preprogrammed infusion of alfentanil to constant arterial plasma concentration,” Anesth. Analg. vol, 76, pp. 556-561, 1993.

[9] D. P. Crankshaw, F. Karasawa, "A method for implementing programmed infusion of thiopentone and methohexitone with a simple infusion pump,” Anaesth. Intensive Care, vol. 17, pp 496-499, 1989.

[10] D. P. Crankshaw, "Intravenous delivery systems: Australian Perspective,” In Intravenous Anaesthesia. Ed. P. F White, Williams \& Wilkins, Philadelphia. 1997, pp 539-544. 